\title{
Article \\ Overview of Intelligent Vehicle Infrastructure Cooperative Simulation Technology for IoV and Automatic Driving
}

\author{
Zirui Ding * and Junping Xiang
}

check for updates

Citation: Ding, Z.; Xiang, J. Overview of Intelligent Vehicle Infrastructure Cooperative Simulation Technology for IoV and Automatic Driving. World Electr. Veh. J. 2021, 12, 222. https://doi.org/ 10.3390/wevj12040222

Academic Editor: Zonghai Chen

Received: 30 September 2021

Accepted: 5 November 2021

Published: 8 November 2021

Publisher's Note: MDPI stays neutral with regard to jurisdictional claims in published maps and institutional affiliations.

Copyright: (C) 2021 by the authors Licensee MDPI, Basel, Switzerland. This article is an open access article distributed under the terms and conditions of the Creative Commons Attribution (CC BY) license (https:/ / creativecommons.org/licenses/by/ $4.0 /)$.
Lianyungang JARI Electronics Co., Ltd., Lianyungang 222006, China; 18061366327@163.com

* Correspondence: dingzirui@jari.cn

\begin{abstract}
This paper reviews the development of vehicle road collaborative simulation in the new era, and summarizes the simulation characteristics of two core technologies in the field of transportation after entering the era of Intelligent Networking: Internet of Vehicles technology and automatic driving technology. This paper analyzes and compares the mainstream Internet of Vehicles (IoV) simulation and automatic driving simulation platforms on the market, deeply analyzes the model-based IoV simulation, and explores a new mode of IoV simulation in the era of big data. According to the latest classification standard of automatic driving in 2020, we summarize the simulation process of automatic driving. Finally, we offer suggestions on the development directions of intelligent network-connected vehicle simulation.
\end{abstract}

Keywords: Internet of Vehicles simulation; automatic driving simulation; simulation software

\section{Introduction}

Traffic congestion is a kind of urban traffic phenomenon that refers to a large number of vehicles crowded on a certain section of the traffic road network, causing the traffic flow of this section to be slow or even stagnant. In the past few decades, with the rapid development of the national economy, the number of urban vehicles has increased steadily and substantially. Due to the slow progress of urban planning and the paving of new roads, the urban road network has become saturated, and traffic congestion is an urban traffic phenomenon that often occurs. The excessive frequency of this phenomenon has attracted the attention of experts and scholars at home and abroad. The question of how to solve the problem of urban traffic congestion has become a growing concern in most urban areas around the world.

The intelligent networked vehicle, which integrates IoV technology and autonomous driving technology, is an answer to the problem of traffic congestion. The intelligent networked vehicle is also the main trend of the future development of the automotive industry.

IoV technology refers to the realization of the information connection between vehicles and other vehicles or road infrastructure, with the help of network communication technology on the basis of vehicle perception. At present, there are two common technology protocols: DSRC and C-V2X.V2X simulation is used to simulate the overall traffic network formed by pedestrians, vehicles, and the road infrastructure by connecting traffic simulation and network simulation in different ways.

Autonomous driving is a technology that uses various sensors to collect surrounding environment information as input, and utilizes artificial intelligence models to make decisions and control vehicles semi-automatically or fully automatically. The key technology is environment perception technology and decision-making technology.

The intelligent networked vehicle uses IoV technology to connect with roadside equipment, edge servers, and other sensor devices, in order to analyze and process relevant data and realize information interaction. Intelligent networked vehicles can effectively cope with the complex urban road network conditions and actively reduce or avoid traffic jams. 
For intelligent connected vehicles, the traditional real-vehicle test approaches involve a $\log$ of variable factors, high experimental costs, and critical environmental requirements. On the contrary, for vehicle-road coordination-based traffic simulation, the parameters can be adjusted accordingly and it can provide more feasible solutions for traffic management. Traffic simulation technology based on vehicle-road coordination is an important method and tool for the reconstruction and prediction of traffic information, and it also plays an important role in the management, control, and optimization of the traffic flow. Meanwhile, the simulation data can be reused and the experimental results are reproducible. This technology is helpful for researchers to discover the deficiencies of intelligent transportation systems, speed up the scientific research process, and promote social development. Vehicleroad coordination-based traffic simulation is used to simulate the four aspects of people, vehicles, roads, and communications. Therefore, this technology can be divided into two parts: IoV simulation and autonomous driving simulation.

\section{IoV Simulation Technology}

IoV technology is a multi-domain comprehensive technology that is essentially a mobile self-organizing network. It uses vehicles as network nodes and forms a network by connecting vehicles and the road infrastructure with a specific wireless signal frequency band. This technology can collect and transmit information from OBU and RSU to complete the information interaction between vehicles and the road infrastructure.

However, the complexity of the IoV network structures comes from the high number of nodes and the free movement mode, which makes it difficult to repeat the experiment in real situations and determine the accuracy of actual vehicle tests. Thus, simulation technology is adopted to carry out simulation experiments [1].

IoV simulation is mainly divided into three steps: the first is to simulate the traffic vehicle and obtain the real vehicle motion state and trajectory; the second is to simulate the network, establish the wireless network topology structure with the vehicle as the node, and complete the functions of data transmission, background load, and channel packet level simulation [2]; the third is to couple the vehicle simulation and network simulation. In order to realize the simulation environment, according to different vehicle models and network protocols, simulators are coupled and interacted to form different types of vehicle network simulation platforms.

\subsection{Traffic Simulations}

Traffic simulation is mainly divided into two parts: the simulation of the vehicle motion state and the simulation of the vehicle trajectory. In the simulation process, the position and motion state of high-speed moving vehicles on the topology map at any time significantly influence the network structure. In the actual simulation test, the selection of vehicle model is then particularly important. Precise model selection can increase the confidence level of the simulation [3].

\subsubsection{Traffic Flow Model}

According to different levels of detailed information required by simulation, models can be roughly divided into two categories: macroscopic models and microscopic models.

Macroscopic models first appeared in the 1950s. Lighthill et al. [4,5] formally introduced the knowledge of fluid mechanics into traffic flow theory and established the famous LWR model based on fluid mechanics; thus, this model is also known as the traffic flow fluid mechanics model. The macroscopic model is based on the distribution of traffic flow and initial vehicles, which reflects the overall characteristics of vehicles on the road network. In general, the road network fleet is regarded as a continuous fluid, and the relevant theoretical knowledge of fluid dynamics is used for calculation. Commonly used parameters include traffic flow, average speed, vehicle distribution density, and so on. The macroscopic simulation model architecture is shown in Figure 1. 


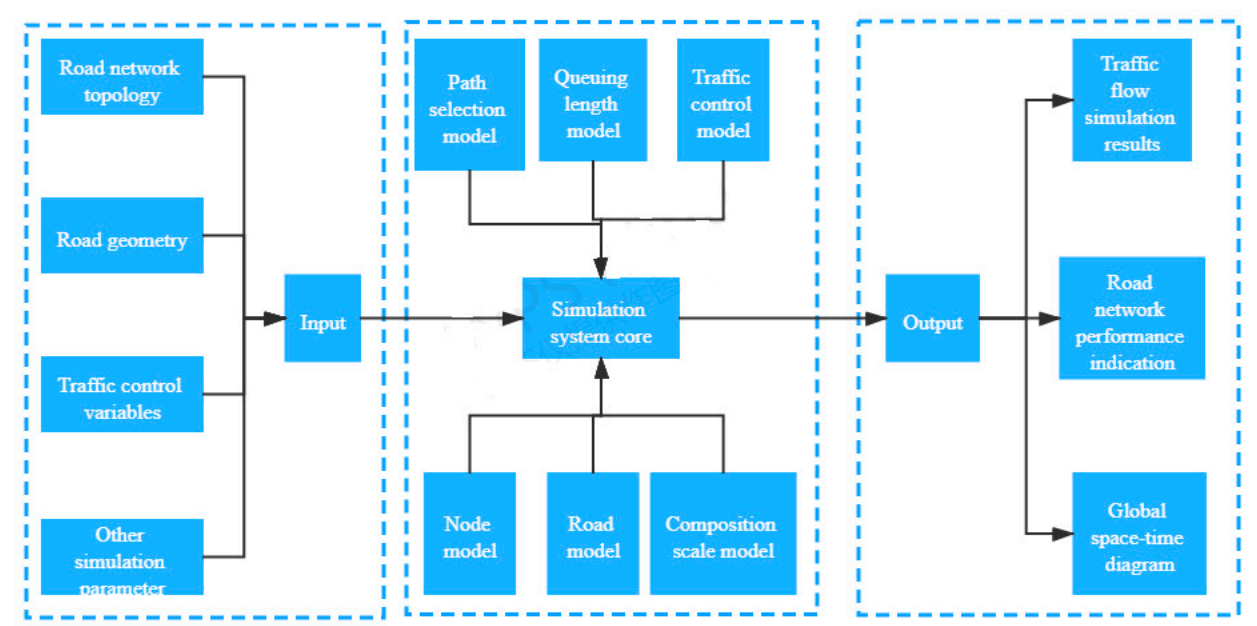

Figure 1. Macroscopic traffic model simulation framework.

The microscopic model is used to model each vehicle as a relatively independent road network object, which can reflect the motion relationship and mutual influence between vehicles. In the study of microscopic models, two models are widely used: the following model and lane changing model. The former can reflect real traffic phenomena, and the latter can evaluate vehicle safety. Commonly used parameters are the absolute position, relative position, speed, acceleration, and so on.

The car-following model is one of the most fundamental parts of microscopic simulation. In this model, vehicles run on a fixed lane and follow other vehicles, and vehicles can interact with each other. Pipes [6] first proposed a simple vehicle following model in the 1950s according to local traffic laws, and afterwards, new vehicle following models were continuously proposed by researchers in the following decades. Through the study of a car-following model, the vehicle speed and speed change can be obtained, and the vehicle position can be updated, which lays the foundation for the following vehicle overtaking, formation, directing, and other motion models.

The vehicle lane change model is established on the basis of a car-following model. It refers to a model in which a vehicle changes lanes under the influence of other vehicles in the current and adjacent lanes. The original vehicle lane change model was proposed by Gipps PG [7] in 1986. According to the urban multi-lane rules, the vehicle lane change model was established by adopting hierarchical decision processing, which provides the theoretical basis and thinking framework for subsequent research on the lane change model. Through the collection, judgment, processing, and command execution of the surrounding information, the driver can avoid the failure of manual lane change due to information error and reduce the probability of traffic accidents.

\subsubsection{Vehicle Trajectory Model}

In the simulation platform, the vehicle trajectory model is composed of different movement constraints, which can be roughly divided into a completely random motion model [8], certain restricted movement model [9], and real trajectory model.

In the completely random motion model, vehicles are treated as nodes and their direction, speed, destinations, and other parameters are completely random. It is the simplest motion model in vehicle simulation. According to the literature [10], this model is rarely used in the environment of IoV because the node distribution is not uniform, and it cannot describe the group movement and is unable to reflect the real vehicle movement mode.

There are two kinds of constraints that affect the motion model: time and space. The motion state of the vehicle at the previous moment affects the motion of the vehicle at the next moment. The movement of surrounding vehicles has an impact and influence on the movement of vehicles, so the restricted movement model can be divided into a time-restricted movement model and space-restricted movement model. 
In the early stage, due to technical limitations, the mobility models used in vehicle network simulation were generally the random model and restricted mobility model mentioned previously. With the improvement of network technology, it has been proven that the accuracy of simulation can be improved significantly with the help of a real trajectory model [11].

The real trajectory model refers to the pre-recording of the trajectory node data of the vehicle in the actual movement, and each recorded node is reflected in the simulation process of the IoV as an independent event. The advantage is to reproduce the real movement of vehicles and the relationship between vehicles. The disadvantage is that, due to the difficulty in obtaining a large number of available trajectories, it can only be used in a local scope and cannot be applied to the whole road network, and the simulation performance is lower than that of other models [12]. The conceptual framwork of real vehicle movement model is shown in Figure 2.

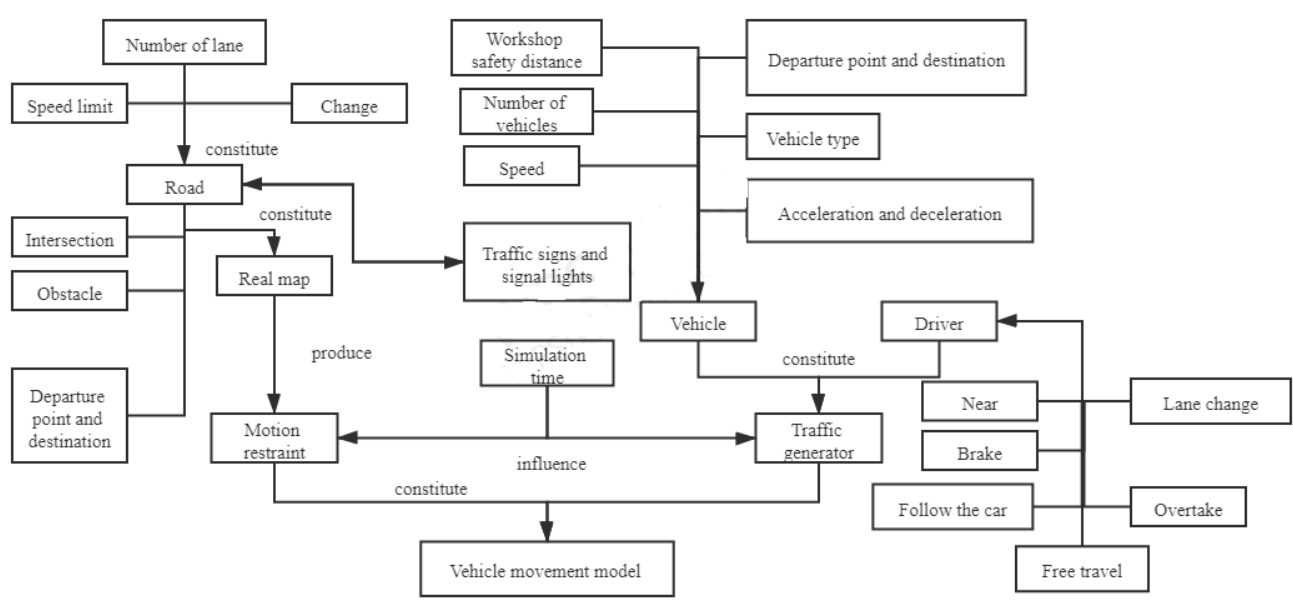

Figure 2. Conceptual framework of real vehicle movement model.

\subsection{Network Simulation}

Network communication simulation is mainly used to obtain network parameters by constructing a mathematical model and performing the simulation of the real road network state and date transmission behavior. Based on network simulation, researchers can control the cost of IoV simulation and flexibly change the simulation framework according to different research needs, which is beneficial for detecting the existing problems of the system and changing the relevant algorithms and functions.

In the real world, the quality of the wireless communication network between vehicles will be affected by many factors, such as the shielding and reflection of building walls, the influence of adjacent vehicles, and the multi-path effect. Thus, it is necessary to simulate the wireless communication channel between vehicle and road. Common communication channel models include the free space propagation model [13], double ray reflection model [14], shadowing model [15], and channel model adopted by the IEEE 802.11p working group [16].

The ideal signal propagation model is the free space propagation model, which is also one of the most widely used channel models in the field of simulation. However, since there is no propagation space, with no attenuation, no obstruction, and no multi-path in real life, this model cannot reflect the real situation and can only be used for theoretical research.

The shading model was proposed by considering the shading effect of a signal due to buildings or obstacles. There are two common methods of constructing a shading model: one is to realize it by constructing a random model; the other is to realize it by constructing a geometric model.

The most widely used model for commercial application is the channel model adopted by the 802.11 p working group. This model is based on the linear delay tap model and 
takes into account both the time domain and the frequency domain. Combining a tap with multiple paths makes it closer to reality and considers a comprehensive range of scenarios. However, the non-stationarity and multi-path correlation between the channels are not considered.

Compared with the free space propagation model, the double ray reflection model, which considers the ground-reflected radio signal, is more practical. The model can predict the path loss between the receiver and the transmitter, so that the vehicle data can be tracked by a fitting ray.

\subsection{IoV Simulation Platform}

According to the different connection methods between the traffic simulator and the network simulator, the existing mainstream car networking platforms can be roughly divided into the following two types: joint type and integrated type.

\subsubsection{Joint Simulation Platform}

The joint vehicle networking simulation platform refers to the use of interfaces to create a simulation environment that connects the traffic simulator and the network simulator. The advantage is that the existing simulator can be used, but the disadvantage is that the interface design is complicated and difficult to realize.

Veins [17] is a joint IoV simulation platform with an open-source framework design. The network simulator is developed based on OMNeT++, and the traffic simulator is developed based on SUMO. OMNeT++ is a free and open source multi protocol network simulation software. Its full English name is objective modular network testbed in $\mathrm{C}++$. SUMO is an open source, highly portable, microscopic and continuous multi-modal traffic simulation package designed to handle large networks. The future development and road-map of SUMO is shaped and fostered by the open-Mobility Working Group at the Eclipse Foundation. The software has a fast data processing speed and is mostly used for large-scale traffic simulation. The protocols supported are IEEE 802.11p, IEEE 1690.4, and cellular network model libraries. Users can expand the software according to their own needs.

Corsim is a traffic simulation software developed by the US Federal Highway Administration. It is the result of combining the NETSIM model for ground street simulation and the FRESIM model for highway simulation. It can simulate not only complex geometric sections but also the interaction between different traffic network components.

TraNS [18] is an open-source application based on Java. The network simulator is developed based on NS-2, and the traffic simulator is developed based on SUMO. The platform has two modes of operation: one is a network program-centrist mode, which can evaluate the real-time mobility of vehicles with the help of network programs; the other is an application-centrist mode, which can evaluate vehicle behaviors with the aid of application.

\subsubsection{Integrated Simulation Platform}

The integrated IoV simulation platform combines the network and traffic simulators in a single simulator. The advantage is that the simulation efficiency is higher, but the disadvantage is that the development is more difficult.

VSimRTI [19] is an integrated IoV simulation platform. Through effective management of the connected simulators, it realizes synchronization, mutual communication, and work cycle correction between multiple simulators. The supported protocol is the standard IEEE. The coupling simulator can increase the versatility of replacing the simulator, and can also evaluate the data communication scheme and algorithm protocol of the network.

TransModeler is a traffic simulation software developed by Caliper, USA. It can provide macro, meso, and micro hybrid simulation. This software enables high-precision microscopic simulation to be integrated with mesoscopic and macroscopic simulations of any road section under the same road network. TransModeler has an intuitive user 
interface and simple operation. It is also the only simulation running software that can be fully supported by the Windows standard.

NCTUns is a distributed and open network simulator, which can directly use the real TCP/IP protocol stack [20] and has a strong visualization effect. It consists of eight [21] mutually independent functional parts: a graphical user interface, simulation task coordinator, modified kernel, protocol daemon, and real application.

In summary, the current commonly used simulation platforms for IoV are compared and analyzed, and the results are shown, in Table 1 below.

Table 1. Comparison of typical Internet of Vehicles simulation platforms.

\begin{tabular}{|c|c|c|c|c|c|c|}
\hline Simulation Platform & Veins & Corsim & TraNS & VSimRTI & TransModeler & NCTUns \\
\hline Open source & Yes & No & Yes & Yes & Yes & Yes \\
\hline Real map & Support & No Support & Support & Support & Support & Support \\
\hline Occlusion model & Support & Support & No support & Support & Support & Support \\
\hline Emission model & No support & No Support & No support & Support & No Support & No support \\
\hline Channel simulation & Support & No support & Support & No support & No Support & No support \\
\hline Large-scale simulation & Support & Support & No support & Support & Support & Support \\
\hline Bit-level simulation & No & No & Yes & No & Yes & No \\
\hline $\begin{array}{l}\text { Wireless network } \\
\text { communication } \\
\text { protocol }\end{array}$ & $\begin{array}{l}\text { LIE/DSRC } \\
\text { WAVE } \\
\text { IEE.802.11p }\end{array}$ & $\begin{array}{l}\text { WiMAX } \\
\text { IEEE.802.11p }\end{array}$ & IEEE.802.11p & $\begin{array}{c}\text { LIE } \\
\text { IEEE.802.11p }\end{array}$ & IEEE.802.11p & $\begin{array}{c}\text { WiMAX/DSRC } \\
\text { WAVE } \\
\text { IEEE.802.11p }\end{array}$ \\
\hline
\end{tabular}

\section{Autonomous Driving Simulation Technology}

With the new round of technological revolution and industrial transformation, autonomous vehicles have become the strategic direction for the development of intelligent networked vehicles.

As early as 2016, the International Society of Automotive Engineers (SAE) [22] divided driving into six levels according to the ability of autonomous vehicles to operate without human intervention: from level 0 (without automation) to level 5 (full unlimited automation). The details are described in Table 2.

Table 2. Level of driving automation proposed by SAE [23].

\begin{tabular}{|c|c|c|c|c|c|c|}
\hline \multirow[b]{2}{*}{ Level } & \multirow[b]{2}{*}{ Name } & \multirow[b]{2}{*}{ Narrative Definition } & \multicolumn{2}{|l|}{ DDT } & \multirow[b]{2}{*}{ DDT Fallback } & \multirow[b]{2}{*}{ ODD } \\
\hline & & & $\begin{array}{c}\text { Sustained Lateral } \\
\text { and Longitudinal } \\
\text { Vehicle Motion } \\
\text { Control }\end{array}$ & OEDR & & \\
\hline \multicolumn{7}{|c|}{ Driver performs part or all of the DDT } \\
\hline 0 & $\begin{array}{l}\text { No Driving } \\
\text { Automation }\end{array}$ & $\begin{array}{l}\text { The performance by the driver of the } \\
\text { entire DDT, even when enhanced by } \\
\text { active safety systems. }\end{array}$ & Driver & Driver & Driver & $\mathrm{n} / \mathrm{a}$ \\
\hline 1 & $\begin{array}{c}\text { Driver } \\
\text { Assistance }\end{array}$ & $\begin{array}{l}\text { The sustained and ODD-specific } \\
\text { execution by a driving automation } \\
\text { system of either the lateral or the } \\
\text { longitudinal vehicle motion control } \\
\text { subtask of the DDT (but not both } \\
\text { simultaneously) with the expectation } \\
\text { that the driver performs the remainder } \\
\text { of the DDT. }\end{array}$ & Driver and System & Driver & Driver & Limited \\
\hline 2 & $\begin{array}{l}\text { Partial } \\
\text { Driving } \\
\text { Automation }\end{array}$ & $\begin{array}{l}\text { The sustained and ODD-specific } \\
\text { execution by a driving automation } \\
\text { system of both the lateral and } \\
\text { longitudinal vehicle motion control } \\
\text { subtask of the DDT with the expectation } \\
\text { that the driver completes the OEDR } \\
\text { subtask and supervises the driving } \\
\text { automation system. }\end{array}$ & System & Driver & Driver & Limited \\
\hline
\end{tabular}


Table 2. Cont.

\begin{tabular}{|c|c|c|c|c|c|c|}
\hline \multirow[b]{2}{*}{ Level } & \multirow[b]{2}{*}{ Name } & \multirow[b]{2}{*}{ Narrative Definition } & \multicolumn{2}{|l|}{ DDT } & \multirow[b]{2}{*}{ DDT Fallback } & \multirow[b]{2}{*}{ ODD } \\
\hline & & & $\begin{array}{c}\text { Sustained Lateral } \\
\text { and Longitudinal } \\
\text { Vehicle Motion } \\
\text { Control }\end{array}$ & OEDR & & \\
\hline \multicolumn{3}{|c|}{ ADS (“System") performs the entire DDT (while engaged) } & & & & \\
\hline 3 & $\begin{array}{l}\text { Conditional } \\
\text { Driving } \\
\text { Automation }\end{array}$ & $\begin{array}{l}\text { The sustained and ODD-specific } \\
\text { performance by an ADS of the entire } \\
\text { DDT with the expectation that the DDT } \\
\text { fallback-ready user is receptive to } \\
\text { ADS-issued requests to intervene, as } \\
\text { well as to DDT performance-relevant } \\
\text { system failures in other vehicle systems, } \\
\text { and will respond appropriately. }\end{array}$ & System & System & $\begin{array}{c}\text { Fallback-ready } \\
\text { user (becomes } \\
\text { the driver } \\
\text { during fallback) }\end{array}$ & Limited \\
\hline 4 & $\begin{array}{l}\text { High Driving } \\
\text { Automation }\end{array}$ & $\begin{array}{l}\text { The sustained and ODD-specific } \\
\text { performance by an ADS of the entire } \\
\text { DDT and DDT fallback without any } \\
\text { expectation that a user will respond to a } \\
\text { request to intervene. }\end{array}$ & System & System & System & Limited \\
\hline 5 & $\begin{array}{l}\text { Full Driving } \\
\text { Automation }\end{array}$ & $\begin{array}{l}\text { The sustained and unconditional (i.e., } \\
\text { not ODD-specific) performance by an } \\
\text { ADS of the entire DDT and DDT } \\
\text { fallback without any expectation that a } \\
\text { user will respond to a request to } \\
\text { intervene. }\end{array}$ & System & System & System & Unlimited \\
\hline
\end{tabular}

On 9 March 2020, the Ministry of Industry and Information Technology issued the "Autonomous Driving Classification" [24] according to the national standard formulation and revision plan, which divided driving automation into $0-5$ levels, a total of six levels. This indicates that China has an independent autonomous vehicle classification standard, laying the foundation for subsequent research. The details are described in Table 3.

Table 3. Taxonomy of driving automation.

\begin{tabular}{|c|c|c|c|c|c|c|}
\hline Level & Level 0 & Level 1 & Level 2 & Level 3 & Level 4 & Level 5 \\
\hline Name & $\begin{array}{l}\text { Emergency } \\
\text { assistance }\end{array}$ & $\begin{array}{l}\text { Partial driving } \\
\text { assistance }\end{array}$ & $\begin{array}{l}\text { Combined driving } \\
\text { assistance }\end{array}$ & $\begin{array}{l}\text { Conditional } \\
\text { automation }\end{array}$ & $\begin{array}{l}\text { High } \\
\text { automation }\end{array}$ & $\begin{array}{c}\text { Full } \\
\text { automation }\end{array}$ \\
\hline $\begin{array}{l}\text { Narrative } \\
\text { Definition }\end{array}$ & $\begin{array}{l}\text { The driving } \\
\text { automation } \\
\text { system cannot } \\
\text { continuously } \\
\text { perform the } \\
\text { lateral or } \\
\text { longitudinal } \\
\text { motion control } \\
\text { of the vehicle in } \\
\text { the dynamic } \\
\text { driving task, but } \\
\text { it has the ability } \\
\text { to continuously } \\
\text { perform the } \\
\text { detection and } \\
\text { response of } \\
\text { some targets and } \\
\text { events in the } \\
\text { dynamic driving } \\
\text { task }\end{array}$ & $\begin{array}{l}\text { The driving } \\
\text { automation system } \\
\text { continuously } \\
\text { performs vehicle } \\
\text { lateral or } \\
\text { longitudinal } \\
\text { motion control in } \\
\text { dynamic driving } \\
\text { tasks within its } \\
\text { design operating } \\
\text { conditions, and it } \\
\text { has the ability to } \\
\text { detect and respond } \\
\text { to some targets and } \\
\text { events that are } \\
\text { compatible with } \\
\text { the executed } \\
\text { vehicle lateral or } \\
\text { longitudinal } \\
\text { motion control }\end{array}$ & $\begin{array}{l}\text { The driving } \\
\text { automation system } \\
\text { continuously } \\
\text { performs vehicle } \\
\text { lateral and } \\
\text { longitudinal } \\
\text { motion control in } \\
\text { dynamic driving } \\
\text { tasks within its } \\
\text { design operating } \\
\text { conditions, and it } \\
\text { has the ability to } \\
\text { detect and respond } \\
\text { to some targets and } \\
\text { events that are } \\
\text { compatible with } \\
\text { the executed } \\
\text { vehicle lateral and } \\
\text { longitudinal } \\
\text { motion control }\end{array}$ & $\begin{array}{l}\text { The driving } \\
\text { automation } \\
\text { system } \\
\text { continuously } \\
\text { performs all } \\
\text { dynamic } \\
\text { driving tasks } \\
\text { within its } \\
\text { design } \\
\text { operating } \\
\text { conditions }\end{array}$ & $\begin{array}{l}\text { The driving } \\
\text { automation } \\
\text { system } \\
\text { continuously } \\
\text { performs all } \\
\text { dynamic } \\
\text { driving tasks } \\
\text { and performs } \\
\text { dynamic } \\
\text { driving task } \\
\text { takeover } \\
\text { within its } \\
\text { design } \\
\text { operating } \\
\text { conditions }\end{array}$ & $\begin{array}{l}\text { The driving } \\
\text { automation } \\
\text { system } \\
\text { continuously } \\
\text { performs all } \\
\text { dynamic } \\
\text { driving tasks } \\
\text { and performs } \\
\text { dynamic task } \\
\text { takeover under } \\
\text { any diving } \\
\text { conditions }\end{array}$ \\
\hline
\end{tabular}


The simulation principle of autonomous driving technology is to replace the real-world vehicle controller with a computer algorithm model in the simulation scene constructed by the simulation platform, and to integrate the simulation sensor and other technologies to complete the test of the computer model.

\subsection{Autonomous Driving Simulation Development Steps}

The development of autonomous driving simulation generally includes the following processes.

Construction of virtual simulation scene library: users build simulation scenes according to the functions required by the autonomous vehicle. The scene library consists of two parts: static scenes and dynamic scenes. Static scenes include road elements (traffic lights, lane lines, ground material, etc.), traffic participants (people and vehicles), and road surrounding elements (green belts, buildings, etc.). Dynamic scenarios include motor vehicle simulation, non-motor vehicle simulation, traffic signal control simulation, etc.

Sensor simulation: add corresponding on-board sensors to the simulated virtual vehicle based on the sensors equipped in the real car. At the same time, the various parameters of the sensor are set to be consistent with the actual vehicle. Commonly used sensors include GPS, V2X, cameras, and millimeter-wave radars, etc.

System architecture: import the algorithm into the simulation environment, so that the vehicle meets the requirements of driving in the scene. Since the system requires a large amount of data for simulation training, it has higher requirements on the storage and computing capabilities of the computer. Therefore, a distributed parallel architecture is generally used to allocate tasks and coordinate calculations to improve simulation efficiency.

Test analysis: perform unified and repeated tests on the designed simulation system. According to the test results, verify whether the system can release the test group [25], and, based on the simulation test results, modify the algorithm architecture or adjust various simulation parameters.

\subsection{Autonomous Driving Simulation Platform}

\subsubsection{Prescan}

Prescan [26], developed by TASS International, is a commercial simulation software for autonomous vehicles. The engine used is MATLAB, which is based on ADAS simulation transformation. The simulation of the vehicle dynamics model is quite accurate. It has a comprehensive three-dimensional scene and a flexible interactive interface, which can be integrated with a variety of hardware-in-the-loop test platforms, but the rendering effect is poor.

\subsubsection{AirSim}

AirSim [27] is Microsoft's open-source autopilot simulation platform based on the virtual 4 physics engine. Through the virtual engine plugin, the interaction of the traffic simulation scene can be realized, and a large amount of labeled perceptual data information can be generated at the same time. The realism of the virtual scene is very high, and the designed $\mathrm{C}++/$ Python interface also has many brief introductions, but it requires high development and programming capabilities.

\subsubsection{VTD}

VTD [28] was developed by the German company VIRES. It is a modular simulation platform based on a hardware-in-the-loop system for the development of autonomous driving algorithm systems. Currently, it can only be run on Linux systems. The platform supports a full-cycle development process from SIL to HIL and VIL. Whether it is real-time or non-real-time, stand-alone or high-performance, VTD can be used for simulation. In addition, VTD has high-quality and real-time rendering effects. 


\subsubsection{Vissim}

Vissim is a traffic simulation software developed by the German PTV company. The longitudinal movement of the vehicle uses a psycho-physical car-following model, while the lateral movement of the vehicle uses a rule-based algorithm. With a 2D and 3D graphical interactive interface, it can support offline data statistics and online traffic operation.

\subsubsection{CarSim}

CarSim is a professional system dynamics vehicle behavior simulation software of MSC, developed by two vehicle dynamics experts, Thomas D. Gillespie Michael Sayers and Steve Hann. CarSim simulates and restores real vehicles based on actual vehicle dynamics, and conducts simulation interactions between three-dimensional dynamic vehicles, advanced controllers, and three-dimensional road network environments. It is mostly used for the development and simulation of unmanned vehicle road driving, vehicle dynamics, and vehicle chassis control algorithms.

We compare and analyze the software mentioned in the above article, and the specific contents are described in Table 4.

Table 4. Comparison of autonomous driving simulation software.

\begin{tabular}{|c|c|c|c|c|c|}
\hline Name & Prescan & AirSim & Vissim & VTD & CarSim \\
\hline Open source & Business & Open source & Business & Business & Business \\
\hline Visualization & $\begin{array}{l}\text { Excellent graphical } \\
\text { interactive, } \\
\text { providing a variety } \\
\text { of visual models }\end{array}$ & $\begin{array}{l}\text { High reduction and } \\
\text { rendering ability, } \\
\text { three-dimensional } \\
\text { environment, real light } \\
\text { and shadow effects }\end{array}$ & $\begin{array}{l}2 \mathrm{D} / 3 \mathrm{D} \text { scene } \\
\text { map, general } \\
\text { picture effect }\end{array}$ & $\begin{array}{l}\text { High-precision } \\
\text { real-time image } \\
\text { rendering } \\
\text { capability }\end{array}$ & $\begin{array}{l}\text { Intuitive graphical } \\
\text { user interface, } \\
\text { results can be } \\
\text { presented with 3D } \\
\text { animation }\end{array}$ \\
\hline Vehicle dynamics & Simple & Simple & Simple & Simple & Real reaction \\
\hline $\begin{array}{l}\text { Autopilot } \\
\text { algorithm } \\
\text { verification } \\
\text { suitability }\end{array}$ & Suitable & Suitable & Non-suitable & Suitable & Non-suitable \\
\hline $\begin{array}{c}\text { Professional } \\
\text { degree simulation }\end{array}$ & Ordinary & Moderate & Ordinary & Ordinary & Major \\
\hline Complexity & Simple & Simple & Simple & Simple & Complex \\
\hline $\begin{array}{l}\text { Difficulty of } \\
\text { getting started }\end{array}$ & Easy & Moderate & Moderate & Easy & Harder \\
\hline
\end{tabular}

\section{Simulation Experiment and Results}

Through the analysis and comparison of the above software, four common traffic simulation software programs are selected for more detailed horizontal comparison. Because Vissim is more mature and used more frequently in software engineering, the advantages and disadvantages of the software are more obvious. Therefore, taking Vissim as the reference standard, this paper designs an experiment to simulate the driving state of vehicles under driver-less conditions. The experimental results are compared with the simulation results of Prescan, TransModeler, and Corsim under the same conditions.

\subsection{Experimental Purpose}

Vissim is used to simulate the operation law of intelligent vehicles on the traffic road network, and finally realize the six functions of cruising, following, lane changing, starting and parking, speed change, and obstacle avoidance. The same test code is tested on three different simulation software programs, namely Prescan, TransModeler, and Corsim. Based on the analysis results, the advantages and disadvantages of the four software programs are obtained. 


\subsection{Experimental Steps}

Intelligent vehicle simulation is a complex traffic simulation project that contains a variety of random components and various logical relationships. Only by following certain procedures and steps can we build a simulation system with a complete architecture, correct procedures, and good performance.

\subsubsection{Define the Experimental Objectives}

This experiment is intended to simulate the running state of vehicles on the road network, so it is necessary to simulate the six common driving laws of vehicles in real life, which are: vehicle cruise, following behavior, overtaking and lane changing, vehicle start and stop, acceleration and deceleration, and collision avoidance.

\subsubsection{Establish Simulation Road Network}

The basic simulation road network is constructed by Vissim. The east-west direction contains 8 two-way lanes, two through lanes, one right turn lane, and one left turn lane. The lanes in the north-south direction are 8 two-way lanes. Movements include go straight, turn right into one lane, and turn left into a separate lane. On the east-west lane, there is a bus stop $100 \mathrm{~m}$ away from the intersection parking line, where pedestrians can transfer buses. The road network structure settings of the other three software programs are the same as in Vissim. The road network modeling used in the simulation experiment is shown in Figure 3.

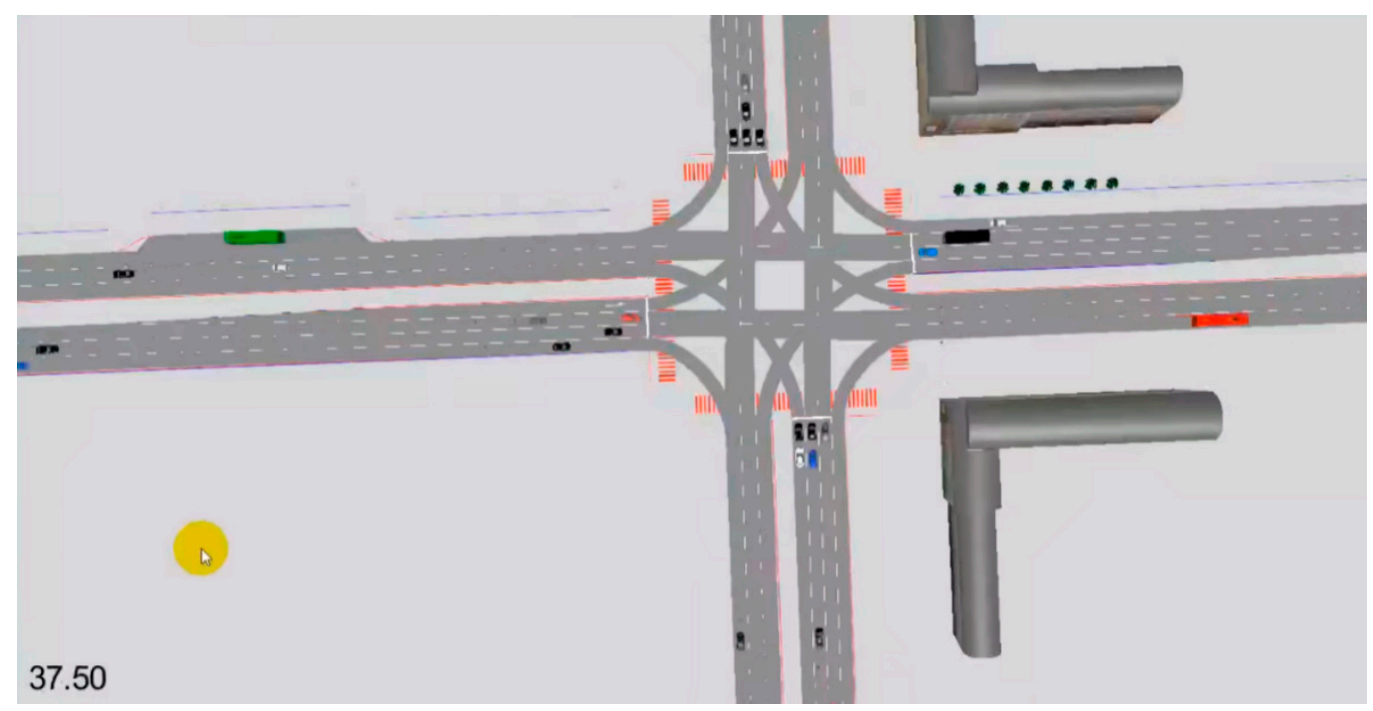

Figure 3. Simulated road network structure.

\subsection{Experimental Scheme and Result Analysis}

The programming language used in this experiment is Java. We use Java to call Vissim, Prescan, TransModeler, and Corsim simulation software for secondary development. The experiment is divided into three parts. The first part is to realize the parking and speed change operation before vehicles arrive at the intersection. When there is congestion at the current intersection, we determine the traffic flow density in advance. If the threshold is exceeded, the lane change operation command is executed independently. We observe the following model, lane changing model, and model spacing of the different simulation software programs. For the second part, under the condition of no traffic congestion, we set the phase cycle of the signal light, change the yellow light time of the signal light, and observe the intersection vehicle behavior of the different simulation software programs. For the third part, when the traffic flow density is large, we set up bus stops in order to observe the impact of bus departures with the different simulation software programs on traffic flow and whether there is a bus priority model. 


\subsubsection{Simulation Scene Effect Analysis}

This experiment uses four different simulation software programs to build the same simulation scene. Due to the different road network processors of the software, the operation and picture presentation quality of the road section structure are also different. With the development of modern society, 3D technology is becoming more and more popular. The scene display of this experiment focuses on observing the visual interaction effect of the 3D interface. The comparison results are shown in Table 5.

Table 5. Comparison of road network construction with different software.

\begin{tabular}{|c|c|c|c|c|}
\hline Name & Vissim & Prescan & TransModeler & Corsim \\
\hline $3 \mathrm{D}$ visualization & $\begin{array}{l}\text { General 3D effect, } \\
\text { GIS-T graphic display }\end{array}$ & $\begin{array}{l}\text { There are many 3D visual } \\
\text { modes, but the scene } \\
\text { rendering effect is general, } \\
\text { with GIS-T graphic display }\end{array}$ & $\begin{array}{l}\text { Excellent 3D effect, } \\
\text { GIS-T graphic display }\end{array}$ & $\begin{array}{l}\text { No 3D effect, no GIS } \\
\text { graphic display }\end{array}$ \\
\hline $\begin{array}{l}\text { Road network } \\
\text { structure }\end{array}$ & $\begin{array}{l}\text { The "line-connection" } \\
\text { structure description is } \\
\text { adopted, and the road } \\
\text { network layout is fine }\end{array}$ & $\begin{array}{l}\text { Modular road network is } \\
\text { adopted to build the road } \\
\text { network, and IBEO scanning } \\
\text { data can also be used to } \\
\text { automatically convert it into } \\
\text { a simulation scene. The road } \\
\text { network layout is } \\
\text { relatively fine }\end{array}$ & $\begin{array}{l}\text { The "node-arc segment" } \\
\text { structure is adopted to } \\
\text { provide an accurate } \\
\text { network collection } \\
\text { editing function, and } \\
\text { the network layout is } \\
\text { relatively fine }\end{array}$ & $\begin{array}{l}\text { The "node-arc segment" } \\
\text { structure is used to } \\
\text { describe the road } \\
\text { network, and the road } \\
\text { geometry is edited by } \\
\text { defining the curvature. } \\
\text { The road network layout } \\
\text { is relatively fine }\end{array}$ \\
\hline
\end{tabular}

\subsubsection{Car-Following Model, Lane Changing Model, and Model Spacing}

During the test of this part of the scheme, two traffic flow states are set: no traffic congestion and traffic congestion. Under different traffic flow conditions, we test the driving state and start-stop changes of vehicles before reaching the intersection, as well as the vehicle spacing when following and changing lanes in the case of traffic congestion ahead. The comparison results are shown in Table 6.

Table 6. Compare the model spacing in different simulation software.

\begin{tabular}{ccccc}
\hline Name & Vissim & Prescan & TransModeler & Corsim \\
\hline Distance between front and rear vehicles when following & $2 \mathrm{~m}-8 \mathrm{~m}$ & $3 \mathrm{~m}-10 \mathrm{~m}$ & $2 \mathrm{~m}-8 \mathrm{~m}$ & $4 \mathrm{~m}$ \\
Distance between front and rear vehicles when changing lanes & $40 \mathrm{~m}$ & $35 \mathrm{~m}$ & $40 \mathrm{~m}$ & $40 \mathrm{~m}$ \\
\hline
\end{tabular}

Since Vissim and TransModeler adopt both a psycho-physiological car-following model and rule-based lane changing model, there is little difference between the front and rear workshop distances during car following and lane changing. Although the lane change spacing of Corsim is the same as that of Vissim and TransModeler software, its car following spacing is basically kept at $4 \mathrm{~m}$. Compared with the other three simulation software programs, the car following distance of Prescan is longer, and the lane changing distance is smaller, which is maintained at approximately $35 \mathrm{~m}$.

\subsubsection{Vehicle Behaviors at Intersection with Yellow Light}

In real life, when a vehicle passes through an intersection, if the signal light turns yellow (especially when the green light turns yellow), the driver usually does not slow down, but accelerates through the intersection. In order to fit the actual vehicle behavior at intersections in China, in the experiment, the car can pass through when the yellow light is on, and we set different yellow light phase times, which are $0 \mathrm{~s}, 2 \mathrm{~s}$, and $5 \mathrm{~s}$, respectively. We observe the vehicle behavior and driver response results of the different simulation software programs. The comparison results are shown in Table 7. 
Table 7. The number of vehicles passing through the intersection with different simulation software.

\begin{tabular}{ccccc}
\hline Name & Vissim & Prescan & TransModeler & Corsim \\
\hline $0 \mathrm{~s}$ & 0 & 0 & 0 & 0 \\
$2 \mathrm{~s}$ & 1 & 1 & 2 & 0 \\
$5 \mathrm{~s}$ & 2 & 2 & 3 & 0 \\
\hline
\end{tabular}

Regardless of how long the phase time of the yellow light is, no vehicle will pass through the intersection when the yellow light is on in the Corsim software. The other three software programs will have vehicles passing through the intersection within $2 \mathrm{~s}$ and $5 \mathrm{~s}$. Vissim and Prescan have the same traffic volume, because both software programs control vehicle behavior based on driver and signal lights, and neither set a variable driver response time. While TransModeler has more vehicles passing through at the same time, it should be a vehicle behavior model with a variable driver response time and special yellow light.

\subsubsection{Bus Stops and Bus Priority}

A special vehicle dynamic priority strategy and special lane layout have been some of the focuses of experts and scholars in the study of traffic flow in recent years. This experiment also tests the related aspects. When the traffic flow density is large, we set up bus stops in order to observe the impact of bus departures with the different simulation software programs on traffic flow and whether there is a bus priority model. The comparison results are shown in Table 8.

Table 8. Comparison of bus systems with different simulation software.

\begin{tabular}{ccccc}
\hline Name & Vissim & Prescan & TransModeler & Corsim \\
\hline $\begin{array}{c}\text { Number of vehicles } \\
\text { queuing at the rear } \\
\text { when leaving the } \\
\text { station }\end{array}$ & 3 & 6 & Can be achieved, the \\
$\begin{array}{c}\text { Implementation of bus } \\
\text { priority strategy }\end{array}$ & $\begin{array}{c}\text { Can be achieved, the } \\
\text { process is simple, and } \\
\text { the implementation } \\
\text { effect is general }\end{array}$ & Not possible & $\begin{array}{c}\text { It can be realized, but the } \\
\text { process is simple, and the } \\
\text { implementation effect is is cumbersome and the } \\
\text { amount of code is huge. Poor } \\
\text { amplementation effect }\end{array}$ \\
\hline
\end{tabular}

In the impact of bus entry and exit on the rear traffic flow, the bus model built in Corsim has the best effect and the minimum number of rear vehicles queuing. Vissim and TransModeler have the same effect. Prescan has a poor effect in the simulation of the bus dynamic priority strategy. The simulation effect of the Corsim software is greatly discounted, the code implementation is too difficult, and the process is cumbersome. Prescan cannot realize the bus priority strategy at all. Vissim and TransModeler can easily complete code editing and realize the bus priority. The difference between them is that the implementation effect of TransModeler is better. To sum up, in this experimental scheme, the comprehensive ability of TransModeler is the best and the ability of Prescan is the worst.

\section{Conclusions}

This article first reviews the development process of vehicle-road collaborative simulation technology, and summarizes the main characteristics of V2X technology and autonomous driving. Then, it analyzes the autonomous driving simulation platform, and conducts an in-depth analysis of the model-driving IoV simulation. According to China's latest autonomous driving classification regulations, the development steps of autonomous driving simulation are analyzed. Finally, based on the discussion and analysis of the commonly used vehicle networking simulation software and automatic driving simulation software in the market, this paper makes the following conclusions: 
1. With the change in the global environment and the increase in extreme weather, the topic of environmental protection has been given more and more attention in many countries. While an building intelligent transportation system, vehicle environmental protection has also become one of the vehicle performance indicators of concern among researchers. After comparing the vehicle networking simulation software involved in this paper, Veins simulation software supports the creation and simulation of a vehicle emission model. Therefore, when the required research involves emission problems, this paper recommends using Veins simulation software for simulation.

2. Secondly, 3D technology is currently a popular technology, which is sought after and utilized in many fields. Having a scene interface with a good 3D effect is the configuration of the current mainstream traffic simulation software, which can enable users to create simulation scenes more intuitively and stereoscopically. Therefore, when 3D models need to be considered during modeling, Corsim software cannot be selected because it does not support a 3D interface. On the contrary, TransModeler, a simulation software, not only supports 3D modeling, but also has an excellent 3D effect.

3. The operation law of domestic traffic flow is different from that of foreign countries in many aspects, especially when the signal light is yellow, as the driver's response and vehicle operation are different. In real life, when a driver encounters a yellow light, he is more inclined to accelerate through the intersection rather than slow down and stop. Therefore, many foreign simulation software programs do not consider this point, such as Corsim, while a few software programs consider this point but do not set a variable driver response model, and the simulation effect is quite different from the actual situation, such as in Vissim and Prescan.

4. One major difference between intelligent vehicles and other traditional vehicles is that intelligent vehicles can avoid collisions independently. The vehicle collects the surrounding environment and vehicle information through the on-board sensor, uploads the original data to MEC for calculation and processing through 5G communication technology, and then transmits the processed auxiliary information back to the vehicle end to form a closed loop. Among the common vehicle networking simulation software, Vissim has a better effect than other simulation software in two aspects: a wireless obstacle model and vehicle lane changing model. Therefore, when the vehicle collision avoidance problem needs to be studied and designed, Vissim simulation software is recommended in this paper.

5. Prescan simulation software adopts a simple vehicle dynamics model in vehicle dynamics, which cannot accurately control the intelligent vehicle in the vertical and horizontal directions, nor can it reflect the dynamic characteristics of the vehicle in the vertical direction. When the required research involves vehicle dynamics, CarSim simulation software is recommended in this paper.

With the in-depth development of network technology, the simulation and optimization development of intelligent networked vehicles based on big data will enter the " $5 \mathrm{~V}$ " era, i.e., Value, Volume, Velocity, Variety, and Veracity.

Author Contributions: Conceptualization, Z.D. and J.X.; methodology, Z.D.; software, Z.D.; formal analysis, Z.D.; investigation, Z.D.; writing-original draft preparation, Z.D.; writing-review and editing, J.X.; supervision, J.X. All authors have read and agreed to the published version of the manuscript.

Funding: This research received no external funding.

Conflicts of Interest: The authors declare no conflict of interest. Both authors are employees of Lianyungang JARI Electronics Co., Ltd. The paper reflects the views of the scientists, and not the company. 


\section{References}

1. Huo, M.; Zheng, Z.; Zhou, X. Simulation of vehicle ad hoc network. Appl. Res. Comput. 2010, 27, $1614-1620$.

2. Martinez, F.J.; Toh, C.K.; Cano, J.-C.; Calafate, C.; Manzoni, P. A survey and comparative study of simulators for vehicular ad hoc networks (VANETs). Wirel. Commun. Mob. Comput. 2009, 11, 813-828. [CrossRef]

3. Shi, C.J.; Ren, Q.H.; Zheng, B. Simulation Research of Node Mobility Models in MANET. Comput. Eng. 2009, 35, 101-103.

4. Lighthill, M.J.; Whitham, G.B. On kinematic waves II. A theory of traffic flow on long crowded roads. Proc. R. Soc. 1955, 299, 317-345.

5. Richards, P.I. Shock Waves on the Highway. Oper. Res. 1956, 4, 42-51. [CrossRef]

6. Pipes, L.A. An Operational Analysis of Traffic Dynamics. J. Appl. Phys. 1953, 24, 274-281. [CrossRef]

7. Gipps, P. A model for the structure of lane-changing decisions. Transp. Res. Part B Methodol. 1986, 20, 403-414. [CrossRef]

8. Bettstetter, C. Smooth is Better than Sharp: A Random Mobility Model for Simulation of Wireless Networks. In Proceedings of the 4th ACM international Workshop on Modeling, Analysis and Simulation of Wireless and Mobile Systems-MSWIM '01 2001, Rome, Italy, 21 July 2001; Technische Universit at Munchen Institute of Communication Networks D-80290; Association for Computing Machinery: New York, NY, USA, 2001.

9. Bettstetter, C.; Wagner, C. The Spatial Node Distribution of the Random Waypoint Mobility Model; Technische Universitat Munchen Institute of Communication Networks D-80290: Munich, Germany, 2002.

10. Ahed, A.; Uthman, B. A Simulation Study: The Impact of Random and Realistic Mobility Models on the Performance of By-pass-AODV in Ad Hoc Wireless Networks. EURASIP J. Wirel. Commun. Netw. 2010, 2010, 1-10.

11. Choffnes, D.R.; Bustamante, F.E. An integrated mobility and traffic model for vehicular wireless networks. In Proceedings of the 2nd ACM International Workshop on Vehicular Ad hoc Networks, Cologne, Germany, 2 September 2005; ACM Press: New York, NY, USA, 2005; pp. 69-78.

12. Gramaglia, M.; Trullols-Cruces, O.; Naboulsi, D.; Fiore, M.; Calderon, M. Mobility and connectivity in highway vehicular networks: A case study in Madrid. Comput. Commun. 2016, 78, 28-44. [CrossRef]

13. Liu, H.L.; Liu, X. Establishment of Wireless Channel Model for Unblocked Scene. J. Hubei Polytech. Univ. 2015, $31,30-34$.

14. Zochmann, E.; Guan, K.; Rupp, M. Two-Ray Models in mmWave Communications. In Proceedings of the 18th International Workshop on Signal Processing Advances in Wireless Communications, Sapporo, Japan, 3-6 July 2017; IEEE Press: Piscataway, NJ, USA, 2017; pp. 1-5.

15. Boban, M.; Vinhoza, T.T.V.; Ferreira, M.; Barros, J.; Tonguz, O.K. Impact of Vehicles as Obstacles in Vehicular Ad Hoc Net-works. IEEE J. Sel. Areas Commun. 2011, 29, 15-28. [CrossRef]

16. Guillermo, A.M.; Mary, A.I. Six Time-and Frequency-Selective Empirical Channel Models for Vehicular Wireless LANs. Veh. Technol. Mag. 2007, 2, 2134-2138.

17. Noori, H. Realistic Urban Traffic Simulation as Vehicular Ad-Hoc Network (VANET) via Veins Framework. In Proceedings of the 12th Conference of Open Innovations Association (FRUCT), Oulu, Finland, 5-9 November 2012; pp. 1-7.

18. Chen, Y.Y.; Jia, J.L.; Fan, B. Vehicle Network Simulation Technology for New Generation Intelligent Transportation Systems. Mob. Commun. 2019, 11, 65-74.

19. Liao, M.D. Research on Simulation Technologies of IoV and Applications on Multi-Car Following. Master's Thesis, Jilin University, Changchun, China, 2020.

20. Wang, L. NCTUns: A New Network Simulation Technology. Comput. Technol. Dev. 2008, 18, 80-82.

21. Wang, S.Y.; Kung, H.T. A Simple Methodology for Constructing Extensible and High-Fidelity TCP/IP Network Simulators. In Proceedings of the IEEE INFOCOM'99, Division of Engineering and Applied Sciences Harvard University, Cambridge, MA, USA, 21-25 March 1999; pp. 1134-1143.

22. SAE International. SAE J3016_201806, Taxonomy and Definitions for Terms Related to Driving Automation Systems for On-Road Motor Vehicles; SAE International: Washington, DC, USA, 2016.

23. SAE Standards News: J3016 Automated-Driving Graphic Update. Available online: https://www.sae.org/news/2019/01/ saeupdates-j3016-automated-driving-graphic (accessed on 4 November 2021).

24. Ministry of Industry and Information Technology. Classification of automobile driving automation Standard release. Robot Tech. Appl. 2020, 2, 3-4.

25. Ye, G. Multi-Target Detection and Tracking Algorithm for Autonomous Driving Car Based on a 3D Lidar in Urban Traffic Environment. Master's Thesis, Beijing Institute of Technology, Beijing, China, 2016.

26. Hendriks, F.; Tideman, M.; Pelders, R.; Bours, R.; Liu, X. Development tools for active safety systems: Prescan and VeHIL. In Proceedings of the 2010 IEEE International Conference on Vehicular Electronics and Safety, Qingdao, China, 15-17 July 2010; pp. 54-58.

27. Shah, S.; Dey, D.; Lovett, C.; Kapoor, A. AirSim: High-Fidelity Visual and Physical Simulation for Autonomous Vehicles. In Field and Service Robotics; Springer: Cham, Switzerland, 2017; Volume 3, pp. 621-635.

28. Laschinsky, Y.; Von Neumann-Cosel, K.; Gonter, M.; Wegwerth, C.; Dubitzky, R.; Knoll, A. Evaluation of an active safety light using virtual test drive within vehicle in the loop. In Proceedings of the 2010 IEEE International Conference on Industrial Technology, Via del Mar, Chile, 14-17 March 2010. 